\title{
Fatores associados à função cognitiva de pacientes com Doença Renal Crônica ${ }^{1}$
}

\author{
Valeska Cristine Serra da Costa Guanaré, Katiúscia Marques de Paulo Maranho, \\ Ana Karina Teixeira da Cunha França, Milady Cutrim Vieira Cavalcante \\ Universidade Federal do Maranhão - UFMA, São Luís, MA, Brasil.
}

\begin{abstract}
Resumo: Introdução: O desempenho cognitivo pode ser comprometido em indivíduos acometidos por doenças incapacitantes como a Doença Renal Crônica (DRC). Objetivo: Avaliar a associação entre variáveis sociodemográficas, econômicas e clínicas com o nível cognitivo de pacientes renais atendidos no Hospital Universitário da Universidade Federal do Maranhão. Método: Trata-se de um estudo transversal realizado com pacientes com idade acima de 20 anos que estavam em tratamento de hemodiálise por no mínimo 6 meses. Utilizou-se um questionário idealizado pelas autoras para coletar características sociodemográficas, econômicas e clínicas. Para avaliação cognitiva, foi utilizado o Mini Exame do Estado Mental. A associação entre as variáveis foi verificada através das análises univariada e multivariada. Resultados: Predominaram homens (52,4\%), casados (59\%), procedentes do interior do Maranhão (67,2\%), com faixa etária entre 40-60 anos (37,7\%), que frequentaram a escola por até 4 anos (34,4\%) e possuíam ocupação que exigia maior sobrecarga física $(49,1 \%)$. Na avaliação cognitiva, $76,9 \%$ apresentaram algum nível de declínio cognitivo, pois pacientes em hemodiálise possuem risco aumentado para déficits cognitivos. Houve associação entre déficit cognitivo e escolaridade inferior a 4 anos $(\mathrm{p}=<0,001)$, faixa etária entre 40 e $60(\mathrm{p}=0,035)$, variáveis de muita influência na pontuação do Mini Mental e associação em possuir de 3 a 4 filhos (p=0,043). Conclusão: Relevante número de pacientes com DRC apresentaram déficit cognitivo. Possuir maior idade, baixa escolaridade e maior número de filhos tiveram influência no estado mental avaliado.
\end{abstract}

Palavras-chave: Insuficiência Renal, Diálise, Cognição.

\section{Factors associated with cognitive function of patients with chronic kidney disease}

\begin{abstract}
Introduction: Cognitive performance can be compromised in individuals affected by debilitating diseases such as chronic kidney disease (CKD). Objective: To evaluate the association between sociodemographic, economic and clinical variables with the cognitive level of kidney patients attending the University Hospital of Federal University of Maranhão. Method: It is a cross-sectional study with patients over age 20 years were undergoing dialysis treatment for at least 6 months. We used a questionnaire designed by the authors to collect demographic, economic and clinical characteristics. For cognitive evaluation we used the Mini-Mental State Examination. The association between variables was assessed through univariate and multivariate analyzes. Results: Men predominated (52.4\%), married (59\%), from the interior of Maranhão (67.2\%), aged between 40-60 years (37.7\%) who attended school for up to four years (34.4\%) and had occupation that required greater physical burden (49.1\%). In cognitive assessment, $76.9 \%$ had some degree of cognitive decline, as hemodialysis patients are at increased risk for cognitive deficits. There was an association between cognitive impairment and schooling less than 4 years $(p=<0.001)$, age group 40-60 ( $p=0.035)$ variables influential in scores of Mini Mental and association have 3-4 children ( $\mathrm{p}=0.043$ ). Conclusion: A significant number of patients with CKD showed evidences of cognitive deficit. Older age, low education, more children are associated with this decline.
\end{abstract}

Keywords: Renal Insufficiency, Dialysis, Cognition.

Autor para correspondência: Valeska Cristine Serra da Costa Guanaré, Hospital Universitário, Universidade Federal do Maranhão, Rua Barão de Itapary, 227, Centro, CEP 65020-070, São Luís, MA, Brasil, e-mail: val.serra.costa@hotmail.com

Recebido em Jul. 10, 2015; $1^{\text {a }}$ Revisão em Set. 15, 2015; 2 ${ }^{\mathrm{a}}$ Revisão em Dez. 6, 2015; $3^{\mathrm{a}}$ Revisão em Jan. 12, 2016 ; Aceito em Jan. $25,2016$. 


\section{Introdução}

As doenças crônicas têm recebido maior atenção dos profissionais de saúde nas últimas décadas. Segundo a Organização Mundial de Saúde em 2003, cerca de $60 \%$ da mortalidade e $46 \%$ do quadro de morbidade no mundo eram atribuídas às doenças crônicas não transmissíveis (KUSUMOTA, 2005). Dentre elas, destaca-se a Doença Renal Crônica (DRC), um diagnóstico sindrômico de perda progressiva e irreversível da função renal, considerado um grande problema de saúde pública (BARROS et al., 2006) que acomete um maior número de pessoas a cada ano.

A DRC é uma doença que não contempla expectativa de cura, mas a manutenção do estado de cronicidade, submetendo o paciente a modalidades terapêuticas de substituição da função renal (MATOS; LOPES, 2009). Dentre estas, a hemodiálise é a mais utilizada no mundo e, no Brasil, estima-se que 100.397 pacientes estavam cadastrados em programa regular de diálise em 2013 (SOCIEDADE..., 2013).

A hemodiálise e a própria condição da doença gera perdas funcionais de vários sistemas corporais (LIMA et al., 2013), impóe restriçóes e limitaçóes aos pacientes (CESARINO et al., 2004) e resulta em alterações nas funções cognitivas (MADERO; GUL; SARNAK, 2008). A função cognitiva é um importante componente para o funcionamento normal diário. A capacidade para entender, aprender e reter conhecimento tem um impacto potencial em muitos aspectos da vida (JASSAL et al., 2008).

Pacientes em hemodiálise têm um risco aumentado para déficit cognitivo (MADERO; GUL; SARNAK, 2008; JASSAL et al., 2008), de modo que somente uma minoria destes tem função cognitiva normal, quando submetidos a testes específicos para os diferentes aspectos da aptidão mental (DAHBOUR; WAHBEH; HAMDAN, 2009).

É importante considerar que a etiologia do déficit cognitivo é multifatorial, mas o caráter crônico e debilitante da DRC juntamente com uma rotina exaustiva de tratamentos pode ser responsável por essa alteração (MURRAY et al., 2006). São vários os fatores de risco para este déficit, tais como paciente com idade elevada, alta prevalência de fatores de risco cardiovasculares, diagnóstico de acidente vascular cerebral, hipertensão arterial, diabetes e elevados níveis de uremia (MURRAY et al., 2006).

Estudos apontam o impacto negativo da doença renal na função cognitiva, revelando a manifestação de distúrbios de memória, dificuldade de planejamento de funçôes, alterações na atenção ou diminuição na velocidade do processamento da informação, inabilidade motora ou na fala (MADERO; GUL; SARNAK, 2008; CONDÉ et al., 2010; BOSSOLA et al., 2011).
Também podem ser citadas a baixa capacidade de concentração, mudanças no comportamento e no humor, distúrbios do sono, além de alteraçóes do estado de alerta e consciência do meio ambiente (RIELLA, 2010).

Verifica-se, desta forma, que alteraçóes cognitivas podem comprometer o funcionamento ocupacional ou social do indivíduo e representar um declínio em relação a um nível anteriormente superior de funcionamento, resultando em perdas da independência e autonomia (REIS et al., 2011).

O desempenho do indivíduo ou a maneira como ele executa uma tarefa está sob a influência de diversas variáveis e segundo o contexto em que está inserido, sendo este determinante para sua participação com sucesso nas atividades diárias (CAVALCANTI; GALVÃO, 2007).

Assim, objetivou-se com este estudo contribuir para o aperfeiçoamento do cuidado prestado a esta população a partir da avaliação da associação entre variáveis demográficas, socioeconômicas e clínicas e a funçáo cognitiva de pacientes submetidos à hemodiálise no Hospital Universitário da UFMA (HUUFMA).

\section{Método}

Estudo transversal realizado com pacientes cadastrados em programa regular de hemodiálise na Universidade Federal do Maranhão (HUUFMA). Fizeram parte deste estudo indivíduos de ambos os sexos, com idade acima de 20 anos e que estavam em tratamento hemodialítico por no mínimo seis meses, após confirmação do diagnóstico da cronicidade da doença. Não foram incluídas pessoas com déficit visual severo, amputação de membros, sequelas neurológicas ou dificuldades de comunicação. Foram elegíveis 75 pacientes e a coleta dos dados ocorreu no período de setembro a novembro de 2013.

Constituíram variáveis de interesse para este estudo: sexo, idade, ocupaçáo, tipo de habitação, números de moradores no domicilio e números de filhos. Além destas, foi verificada a escolaridade, avaliada em anos de frequência à escola e categorizada em menor de quatro anos, entre quatro e oito anos e superior ou igual a nove anos. Também foram identificadas procedência, situaçáo conjugal do paciente e a caracterização de situação econômica segundo Critério de Classificação Econômica Brasileira (CCEB).

Como dados clínicos, consideraram-se tempo de hemodiálise, qualidade da diálise e patologia de base. A adequacidade de diálise é fixada pelas diretrizes do National Kidney Foundation Disease Outcomes Quality Initiative, que recomendam que 
se mantenha um $\mathrm{Kt} / \mathrm{V}$ maior que 1,2. Na fórmula do Kt/V, o (K) é a depuração de ureia do dialisador, multiplicada pelo tempo de tratamento $(\mathrm{t})$ e dividido pelo volume de distribuição de ureia do paciente (V). O indicador de qualidade da diálise é utilizado para quantificar a taxa de redução da ureia e sua depuração (BREITSAMETER et al., 2012) e foi selecionado devido à associaçáo entre elevado índice de uremia e déficits cognitivos (RIELLA, 2010).

A avaliação das funçôes cognitivas foi realizada por meio da aplicaçáo do Mini Exame do Estado Mental - Mini Mental durante a sessão de hemodiálise, considerando o período que os pacientes permaneceram no hospital. Trata-se do instrumento mais utilizado para avaliar a função cognitiva por ser um teste rápido e de fácil aplicação. Contempla vários domínios (orientação espacial, temporal, memória imediata e de evocação, cálculo, linguagem-nomeação, repetição, compreensão, escrita e cópia de desenho) agrupados nas cinco áreas da cogniçẫo: "orientação", "registro", "atenção" e "cálculo", "recuperação" e "linguagem”. Além disso, é um dos poucos testes validados e adaptados para a população brasileira (BRASIL, 2006).

A avaliação é feita a partir da atribuição do valor 1 ou 0 , sendo o escore total de 30 pontos. Como pontos de corte, foram considerados pontuação maior/igual a 24, sugerindo normalidade; menor que 24 e maior/igual a 16, declínio leve; entre 10 e 15, declínio moderado; e menor que 10 , como comprometimento grave (BRASIL, 2006).

As variáveis categóricas foram apresentadas por meio de frequências e porcentagens e as quantitativas por média e desvio padrão (média $\pm \mathrm{DP}$ ). As associaçôes foram testadas por meio do teste quiquadrado. Foi adotado nível de significância de $5 \%$. Os dados foram analisados pelo Stata 10.0.

Este estudo faz parte da Pesquisa intitulada "Funcionalidade de pacientes em tratamento dialítico, São Luís- MA" que foi aprovado pelo Comitê de Ética em Pesquisa do HUUFMA e recebeu financiamento da Fundação de Amparo à Pesquisa e Desenvolvimento Científico do Maranhão.

\section{Resultados}

Dentre os 75 pacientes elegíveis, 11 foram a óbito, 2 pacientes foram submetidos ao transplante renal e 1 foi transferido para outra unidade de diálise, representando uma perda de $18,7 \%$. A amostra final foi de 61 pacientes.

A Tabela 1 apresenta a caracterização demográfica e socioeconômica dos sujeitos deste estudo. Predominaram pacientes casados/união consensual

Tabela 1. Características demográficas e socioeconômicas de pacientes em hemodiálise em um Hospital Universitário, 2014.

\begin{tabular}{lcc}
\hline & Variáveis & Total (n=61) \\
\cline { 2 - 3 } & $\mathbf{N}$ & $\%$ \\
\hline Sexo & 32 & 52,4 \\
$\quad$ Masculino & 29 & 47,6 \\
Feminino & & \\
Faixa etária (anos) & 20 & 32,8 \\
$<40$ & 23 & 37,7 \\
$40-60$ & 18 & 29,5 \\
$\geq 60$ & & 27,9 \\
Procedência & 17 & 67,2 \\
São Luís & 41 & 4,9 \\
Interior do MA & 3 & 34,4 \\
Outros estados & & 32,8 \\
Escolaridade (anos) & 21 & 32,8 \\
$<4$ & 20 & 8,2 \\
$4-8$ & 20 & 18,0 \\
$\geq 9$ & & 49,2 \\
Ocupação & 5 & 4,9 \\
Estudante & 11 & 19,7 \\
Do lar e aposentado & 30 & \\
Ocupações que exigem maior sobrecarga física & 3 & \\
Ocupações que exigem maior sobrecarga cognitiva & 12 & \\
Outras & & \\
\hline
\end{tabular}

*n inferior a 61; CCEB - Critério de Classificação Econômica Brasileira; HU-UFMA - Hospital Universitário da Universidade Federal do Maranhão. 
Tabela 1. Continuação...

\begin{tabular}{lcc}
\hline \multicolumn{1}{c}{ Variáveis } & \multicolumn{2}{c}{ Total (n=61) } \\
\cline { 2 - 3 } & $\mathbf{N}$ & $\%$ \\
\hline Situação conjugal & 36 & 59,0 \\
Casado/União consensual & 23 & 37,7 \\
Solteiro & 2 & 3,3 \\
Separado ou viúvo & & \\
Número de filhos* & 25 & 47,2 \\
$0-2$ & 17 & 32,1 \\
$3-4$ & 11 & 20,7 \\
$\geq 5$ & & \\
Número de moradores no domicílio** & 21 & 46,6 \\
$\leq 3$ & 24 & 53,4 \\
$4-5$ & & \\
CCEB & 4 & 5,5 \\
A-B & 18 & 29,5 \\
C & 39 & 63,9 \\
D-E & & \\
Tipo de habitação & 52 & 11,5 \\
Alvenaria & 7 & 3,3 \\
Taipa & 2 & \\
Adobe & & \\
\hline
\end{tabular}

*n inferior a 61; CCEB - Critério de Classificação Econômica Brasileira; HU-UFMA - Hospital Universitário da Universidade Federal do Maranhão.

(59\%), residindo com 4 a 5 pessoas (53,4\%), em habitação de alvenaria $(85,2 \%)$ e procedentes do interior do Maranhão (67,2\%). Quanto à classificação econômica $63,9 \%$ dos pacientes, apresentaram menor poder de compra de bens e serviços.

A Tabela 2 apresenta as características clínicas dos pacientes. Os resultados apontam adequado índice de eficiência dialítica dos pacientes $(K t / V \geq 1,2)$.

A avaliação da função cognitiva, segundo o Mini Mental, está descrita na Tabela 3. Observou-se que $76,9 \%$ dos pacientes apresentaram algum nível de declínio cognitivo.

A Tabela 4 apresenta a associação entre as características demográficas e socioeconômicas com a função cognitiva. Pacientes em hemodiálise na faixa etária de 40 a 60 anos apresentaram maior prevalência de declínio cognitivo em níveis de leve a moderado, quando comparados com pacientes nas demais faixas etárias $(\mathrm{p}=0,014)$. Escolaridade também esteve associada ao declínio cognitivo. Não foi observada na Tabela 5 associação de declínio cognitivo com as variáveis clínicas estudadas.

\section{Discussão}

Pacientes em hemodiálise possuem maior risco para déficit cognitivo (MADERO; GUL; SARNAK, 2008; JASSAL et al., 2008). Estudos sugerem que
Tabela 2. Características clínicas de pacientes em hemodiálise em um Hospital Universitário, 2014.

\begin{tabular}{lcc}
\hline \multicolumn{1}{c}{ Variável Total (n=61) } & N & $\%$ \\
\hline Tempo em diálise (anos) & & \\
$\geq 1 \mathrm{e}<3$ & 2 & 3,3 \\
$\geq 3 \mathrm{e}<5$ & 24 & 39,3 \\
$\geq 5$ & 35 & 57,4 \\
Doença de base & & \\
$\quad$ Hipertensão & 23 & 37,7 \\
Diabetes mellitus & 14 & 22,9 \\
Glomerulonefrite & 10 & 16,4 \\
Outras & 12 & 19,7 \\
Indeterminada & 2 & 3,3 \\
Kt/V & & \\
$\geq 1,2$ & 60 & 98,4 \\
$<1,2$ & 1 & 1,6 \\
\hline
\end{tabular}

$\mathrm{Kt} / \mathrm{V}$ - Qualidade da diálise.

Tabela 3. Função cognitiva segundo o Mini Exame do Estado Mental de pacientes em hemodiálise em um Hospital Universitário, 2014.

\begin{tabular}{lcc}
\hline \multicolumn{1}{c}{ Nível Cognitivo } & $\mathbf{N}$ & $\%$ \\
\hline Normalidade & 36 & 59,1 \\
Declínio leve & 22 & 36,0 \\
Declínio moderado & 3 & 40,9 \\
Declínio grave & 0 & 0,0 \\
\hline
\end{tabular}

a prevalência deste déficit varia de $40 \%$ a $60 \%$ em pacientes renais crônicos em diferentes estágios da DRC, definido pelo Mini Mental (PEREIRA et al., 2007). No estudo de Condé et al. (2010), pacientes 
Tabela 4. Associação entre as características demográficas e socioeconômicas e a função cognitiva no Mini Exame do Estado Mental de pacientes em hemodiálise em um Hospital Universitário, 2014.

\begin{tabular}{|c|c|c|c|c|c|}
\hline \multirow{3}{*}{ VARIÁVEIS } & \multicolumn{4}{|c|}{ Mini Mental } & \multirow{3}{*}{ P-valor } \\
\hline & \multicolumn{2}{|c|}{ Declínio Leve/Moderado } & \multicolumn{2}{|c|}{ Normal } & \\
\hline & $\mathbf{N}$ & $\%$ & $\mathbf{N}$ & $\%$ & \\
\hline \multicolumn{6}{|l|}{ Faixa etária (anos) } \\
\hline$<40$ & 3 & 12,0 & 17 & 47,2 & \\
\hline $40-60$ & 13 & 52,0 & 10 & 27,8 & 0,014 \\
\hline$\geq 60$ & 9 & 36,0 & 9 & 25,0 & \\
\hline Sexo & & & & & 0,952 \\
\hline Masculino & 13 & 52,0 & 19 & 52,8 & \\
\hline Feminino & 12 & 48,0 & 17 & 47,2 & \\
\hline Procedência & & & & & 0,472 \\
\hline São Luís & 5 & 20,0 & 12 & 33,3 & \\
\hline Interior do MA & 19 & 76,0 & 22 & 61,1 & \\
\hline Outros estados & 1 & 4,0 & 2 & 4,5 & \\
\hline Situação conjugal & & & & & 0,616 \\
\hline Casado/União estável & 16 & 64,0 & 20 & 55,6 & \\
\hline Solteiro & 8 & 32,0 & 15 & 41,7 & \\
\hline Separado ou viúvo & 1 & 4,0 & 1 & 2,8 & \\
\hline \multicolumn{6}{|l|}{ Escolaridade (anos) } \\
\hline$<4$ & 15 & 60,0 & 6 & 16,7 & $<0,001$ \\
\hline $4-8$ & 9 & 36,0 & 11 & 30,5 & \\
\hline$\geq 9$ & 1 & 4,0 & 19 & 52,8 & \\
\hline Ocupação & & & & & 0,139 \\
\hline Estudante & 0 & 0,0 & 5 & 13,9 & \\
\hline Do lar e aposentado & 3 & 13,0 & 6 & 16,7 & \\
\hline Exige maior sobrecarga física & 16 & 69,7 & 14 & 38,9 & \\
\hline Exige maior sobrecarga cognitiva & 1 & 4,3 & 2 & 5,6 & \\
\hline Outras & 3 & 13,0 & 9 & 25,0 & \\
\hline Moradores no domicílio & & & & & 0,694 \\
\hline 1 & 8 & 32,0 & 13 & 36,1 & \\
\hline 2 & 9 & 36,0 & 15 & 41,7 & \\
\hline 3 & 8 & 32,0 & 8 & 22,2 & \\
\hline \multicolumn{6}{|l|}{ Número de filhos } \\
\hline $0-2$ & 6 & 27,3 & 19 & 61,3 & \\
\hline $3-4$ & 9 & 40,9 & 8 & 25,8 & 0,043 \\
\hline$\geq 5$ & 7 & 31,8 & 4 & 12,9 & \\
\hline Tipo de habitação & & & & & 0,220 \\
\hline Alvenaria & 20 & 80,0 & 32 & 88,9 & \\
\hline Taipa & 3 & 12,0 & 4 & 11,1 & \\
\hline Adobe & 2 & 8,0 & 0 & - & \\
\hline ССЕВ & & & & & 0,261 \\
\hline A-B & 1 & 4,0 & 3 & 8,3 & \\
\hline $\mathrm{C}$ & 5 & 20,0 & 13 & 36,1 & \\
\hline D-E & 19 & 76,0 & 20 & 55,6 & \\
\hline
\end{tabular}

CCEB - Classificação Econômica Brasil.

em hemodiálise apresentaram pior desempenho neste teste e, neste estudo, $76,9 \%$ dos pacientes apresentaram algum indicativo de declínio cognitivo.

Observou-se associação estatisticamente significante entre declínio cognitivo e faixa etária entre 40 e 60 anos $(\mathrm{p}=0,014)$. Pacientes com escolaridade menor que quatro anos $(\mathrm{p}<0,001)$ e que possuíam 3 filhos ou mais $(\mathrm{p}=0,043)$ também tiveram maior prevalência de declínio cognitivo, ainda que em níveis de leve a moderado.

A escolaridade e a idade são variáveis de muita influência na pontuação do Mini Mental. Estudos realizados em diferentes países demonstraram que, mesmo em indivíduos que náo apresentavam 
Tabela 5. Associação entre as características clínicas e a função cognitiva no Mini Exame do Estado Mental de pacientes em hemodiálise em um Hospital Universitário, 2014.

\begin{tabular}{|c|c|c|c|c|c|}
\hline \multirow{3}{*}{ VARIÁVEIS } & \multicolumn{4}{|c|}{ Mini Mental } & \multirow{3}{*}{ P-valor } \\
\hline & \multicolumn{2}{|c|}{ Declínio Leve/Moderado } & \multicolumn{2}{|c|}{ Normal } & \\
\hline & $\mathbf{n}$ & $\%$ & $\mathrm{n}$ & $\%$ & \\
\hline Tempo em diálise (anos) & & & & & 0,124 \\
\hline$\geq 1 \mathrm{e}<3$ & 1 & 4,0 & 1 & 2,8 & \\
\hline$\geq 3 \mathrm{e}<5$ & 6 & 24,0 & 18 & 50,0 & \\
\hline$\geq 5$ & 18 & 72,0 & 17 & 47,2 & \\
\hline Doença de base & & & & & 0,671 \\
\hline Hipertensão & 11 & 44,0 & 12 & 33,3 & \\
\hline Diabetes mellitus & 6 & 24,0 & 8 & 22,2 & \\
\hline Glomerulonefrite & 2 & 8,0 & 8 & 22,2 & \\
\hline Outras & 5 & 20,0 & 7 & 19,5 & \\
\hline Indeterminada & 1 & 4,0 & 1 & 2,8 & \\
\hline Índice $\mathrm{Kt} / \mathbf{V}$ & & & & & 0,227 \\
\hline$<1,2$ & 1 & 4,0 & 0 & 0,0 & \\
\hline$\geq 1,2$ & 24 & 96,0 & 36 & 100 & \\
\hline
\end{tabular}

$\mathrm{Kt} / \mathrm{V}$ - Qualidade da diálise.

evidências de declínio cognitivo, quanto menor a escolaridade e maior a idade, menor era a pontuaçáo obtida nesta avaliação (TYRRELL et al., 2005; BERTOLUCCI et al., 1994; MURRAY, 2008).

A literatura evidencia que o menor índice de escolaridade está relacionado à maior incidência de casos de doenças crônicas (ISER et al., 2011) e ao pior nível de adesão ao tratamento pelo paciente (COSTA, 2012), pois este fato dificulta o entendimento da enfermidade (LOUREIRO et al., 2011) e pode ocasionar eventuais esquecimentos $\mathrm{da}$ rotina medicamentosa (CAZARINI et al., 2002).

Congruente com dados do último censo da Sociedade Brasileira de Nefrologia (SBN), neste estudo, houve predominância de pacientes submetidos à hemodiálise na faixa etária de 40 a 60 anos (37,7\%) (SOCIEDADE..., 2013) de modo que, conforme estudo desenvolvido em Ribeiráo Preto, parcela significativa dos pacientes em hemodiálise avaliados se tornariam idosos em menos de uma década (KUSUMOTA, 2005).

Houve prevalência de pacientes casados (59,0\%), sendo que a convivência em casal é apontada na literatura como indicativo de suporte familiar, e a rede de apoio estruturada pode ser relacionada à melhoria da adesão ao tratamento de doenças crônicas (ISER et al., 2011; ASSUNÇÃO; URSINE, 2008) e, portanto, a melhores resultados da condição de saúde, minimizando o efeito da baixa escolaridade sob esse aspecto.

A associação entre possuir de 3 a 4 filhos e declínio cognitivo, por sua vez, não está bem retratada na literatura. Segundo Censo de 2011 do Instituto
Brasileiro de Geografia e Estatística, a taxa de fecundidade das mulheres brasileiras com menor nível de escolaridade foi de 3,07 filhos enquanto que mulheres com oito ou mais anos de estudos apresentaram taxa de 1,69 (NIEDERAUER, 2012). A relação entre a escolaridade e declínio cognitivo está bem estabelecida na literatura (BERTOLUCCI et al., 1994; SILVA et al., 2014). Assim, pode-se sugerir que pessoas com menor nível de escolaridade possuem maior número de filhos e isto pode resultar em maior chance de declínio cognitivo.

O comprometimento da função cognitiva também pode ser atribuído ao efeito das toxinas urêmicas, pois o acúmulo de substâncias tóxicas prejudica as funçóes do sistema nervoso central em pacientes renais crônicos (RADIC et al., 2010). Neste estudo, não houve associação estatisticamente significante entre inadequado índice da qualidade de diálise e déficit cognitivo.

As alteraçóes do domínio cognitivo podem ser identificadas a partir de avaliaçôes específicas ou a partir das queixas do paciente quanto a eventuais esquecimentos que estejam interferindo no desempenho de suas atividades (PAULO; YASSUDA, 2010), tais como horários de ingestáo medicamentosa, datas de consultas, dia do tratamento, entre outros. Neste contexto, a melhoria da capacidade cognitiva dos pacientes renais é um dos objetivos da equipe multiprofissional e, dentre eles, do Terapeuta Ocupacional que objetiva também a melhoria do desempenho ocupacional do paciente nas atividades da vida diária e vida prática bem como a reconstrução do cotidiano (TEDESCO; FERRARI, 2000). 
Dentre as estratégias utilizadas por este profissional, podem ser citadas as atividades cognitivas (exercícios para atenção mantida, focada e alternada, orientação espaço-temporal, treino dos diferentes tipos de memória, treino de sequência e planejamento, raciocínio lógico, leitura e aprendizagem), adaptação e simplificação de tarefas, estratégias externas compensatórias como uma nova forma de desempenhar uma atividade utilizando dispositivo externo a fim de permitir ao paciente vivenciar a rotina de maneira mais funcional e independente. Nesse sentido, podem ser utilizadas agendas para anotação de compromissos, alarmes do telefone celular para lembrar o que deve ser feito ou que medicamento ingerir e manter roteiros com as atividades do dia (AMODEO et al., 2010; GINDRI et al., 2012).

Outro enfoque necessário é a adesão dos pacientes renais ao tratamento que podem ser trabalhados por meio de atividades grupais com esclarecimentos acerca do funcionamento da memória e suas peculiaridades. Segundo Tavares et al. (2012), a abordagem grupal constitui não apenas um espaço para abordar aspectos psicológicos, mas também fornece uma rede social de apoio e um espaço educativo capaz de promover esclarecimentos e educação em saúde.

A educação em saúde é de responsabilidade de toda a equipe multiprofissional, inclusive do terapeuta ocupacional. Para isso, deve-se utilizar uma linguagem simples e adequada ao nível sócio-cultural do usuário (CAZARINI et al., 2002) a fim de passar todas as informaçóes necessárias e sanar todas as dúvidas para que as orientaçóes sejam incorporadas pelos pacientes, adotando um comportamento ativo diante de seu tratamento e de seu cotidiano (VIEIRA et al., 2006).

As demais características demográficas, socioeconômicas e clínicas dos sujeitos deste estudo revelaram achado congruente ao do atual perfil de pacientes renais de outros estados e não apresentaram associaçáo com o declínio cognitivo. Houve predomínio de pacientes do sexo masculino $(52,4 \%)$, corroborando com resultados de outros estudos nacionais (LOUREIRO et al., 2011; ZAMBONATO; THOMÉ; GONÇALVES, 2008) e com dados do último censo da SBN (SOCIEDADE..., 2013). Mulheres tendem a procurar mais os serviços de saúde do que homens, revelando uma rotina incorporada de consultas periódicas e maior conscientização dos cuidados de saúde (ASSUNÇÃO; URSINE, 2008). O homem, por sua vez, acredita não ser vulnerável a problemas de saúde, apresentando menos cuidado consigo e, desta forma, maior exposição às situações de risco (SCHRAIBER; GOMES; COUTO, 2005).
Quanto à procedência dos pacientes, identificou-se que $67,2 \%$ advêm do interior do Maranhão, semelhante à situação encontrada no Pará (QUEIROZ; NASCIMENTO, 2006), em que $60 \%$ eram oriundos do interior do Estado realizando tratamento na capital. Estudo sobre a sobrevida de pacientes em diálise no Sistema Único de Saúde do Brasil identificou que a maioria dos pacientes da regiấo sudeste também eram oriundos de cidades do interior do Estado, especialmente de municípios com baixo Índice de Desenvolvimento Humano (SZUSTER et al., 2012). Tal achado retrata a precária situação de saúde em diversos municípios do Brasil, independentemente da região, sendo necessária, muitas vezes, a transferência destes em situação emergencial para os hospitais na capital (QUEIROZ; NASCIMENTO, 2006).

As cidades do interior do Maranhão nem sempre contam com os recursos tecnológicos necessários para o diagnóstico da DRC. Além disso, muitas vezes, há despreparo das equipes de saúde, que tratam o paciente paliativamente por um período longo, enquanto a DRC evolui para estágios mais severos.

$\mathrm{O}$ ambiente no qual o indivíduo é inserido e as atividades realizadas ao longo da vida estão estritamente relacionadas ao desempenho cognitivo (OLIVEIRA et al., 2012). As ocupações exercidas antes dos pacientes deste estudo iniciarem o tratamento hemodialítico estavam, na maioria das vezes, relacionadas a atividades ocupacionais que exigiam maior sobrecarga física $(49,1 \%)$, predominando dentre estas a de lavrador. Esta profissão, por sua vez, é comumente associada à maior dificuldade de acesso ao ensino regular e, consequentemente, à menor escolaridade (SERVIÇO..., 2012).

Em relação ao tempo de diálise, foi observada que a maioria da população estudada realizava hemodiálise por um período igual ou superior a cinco anos (57,4\%). O tempo do tratamento é importante no agravamento das comorbidades e estas têm sido apontadas como determinantes na sobrevida de pacientes em tratamento hemodialítico, mas não possuem relação com o comprometimento da função cognitiva (MORSCH; GONÇALVES; BARROS, 2005; SILVA, 2011), reforçando os achados deste estudo.

Segundo o Censo Brasileiro de Diálise de 2013 (SOCIEDADE..., 2013), as causas primárias da DRC são a hipertensão arterial e a diabetes. Do mesmo modo, no presente estudo, a maioria dos pacientes apresentou como doença de base a hipertensão arterial $(37,7 \%)$ seguida da diabetes $(22,9 \%)$. Em conformidade com a literatura, nenhuma associação entre doença de base e declínio cognitivo foi identificada (SILVA et al., 2014). 


\section{Conclusão}

Um relevante número dos pacientes em tratamento hemodialítico apresentou indicativo de declínio na função cognitiva. Verificou-se associação entre declínio cognitivo e maior idade, menor escolaridade e maior número de filhos.

Esta pesquisa foi relevante para o conhecimento do perfil dos pacientes renais crônicos e as variáveis que contribuem para o declínio cognitivo desta população de modo a contribuir para implementar o cuidado prestado ao doente renal crônico pela equipe multiprofissional, especialmente pelo terapeuta ocupacional que deve considerar os pacientes com maior risco para declínio cognitivo e assim estabelecer um plano terapêutico mais efetivo e adaptado às demandas dos pacientes.

\section{Referências}

AMODEO, M. T.; NETTO, T. M.; FONSECA, R. P. Desenvolvimento de programas de estimulação cognitiva para adultos idosos: modalidades da Literatura e da Neuropsicologia. Letras de Hoje, Porto Alegre, v. 45, n. 3, p. 54-64, 2010.

ASSUNÇÃO, T. S.; URSINE, P. G. S. Estudo de fatores associados à adesão ao tratamento não farmacológico em portadores de diabetes mellitus assistidos pelo Programa Saúde da Família. Ciência \& Saúde Coletiva, Belo Horizonte, v. 13, n. 2, p. 2189-2197, 2008. http://dx.doi. org/10.1590/S1413-81232008000900024.

BARROS, E. J. et al. Nefrologia: rotinas, diagnóstico e tratamento. São Paulo: Artmed, 2006.

BERTOLUCCI, P. H. F. et al. O mini-exame do estado mental em uma população geral: impacto da escolaridade. Arquivos de Neuro-Psiquiatria, São Paulo, v. 52, n. 1, p. 1-7, 1994. http://dx.doi.org/10.1590/S0004$-282 X 1994000100001$.

BOSSOLA, M. et al. Mini Mental State Examination over time in chronic hemodialysis patients. Journal of Psychosomatic Research, Roma, v. 71, n. 1, p. 50-54, 2011. http://dx.doi.org/10.1016/j.jpsychores.2011.01.001. PMid:21665013.

BRASIL. Ministério da Saúde. Envelhecimento e saúde da pessoa idosa. Brasília, 2006. (Cadernos de Atenção Básica).

BREITSAMETER, G. et al. Cálculo de Kt/V em hemodiálise: comparação entre fórmulas. Jornal Brasileiro de Nefrologia, São Paulo, v. 34, n. 1, p. 22-26, 2012.

CAVALCANTI, A.; GALVÃO, C. Terapia Ocupacional: fundamentação \& prática. Rio de Janeiro: Guanabara Koogan, 2007.

CAZARINI, R. P. et al. Adesão a um grupo educativo de pessoas portadoras de diabetes mellitus: porcentagem e causas. Medicina, Ribeirão Preto, v. 35, n. 2, p. $142-$
150, 2002. http://dx.doi.org/10.11606/issn.2176-7262. v35i2p142-150.

CESARINO, C. B. et al. O autocuidado de clientes portadores de hipertensão arterial em um hospital universitário. Arquivos de Ciências da Saúde, São José do Rio Preto, v. 11, n. 3, p. 146-148, 2004.

CONDÉ, S. A. L. et al. Declínio cognitivo, depressão e qualidade de vida em pacientes de diferentes estágios da doença renal crônica. Jornal Brasileiro de Nefrologia, São Paulo, v. 32, n. 3, p. 242-248, 2010.

COSTA, K. P. S. Adesão de pacientes portadores de insuficiência renal crônica à terapia dialítica. 2012. 29 f. Dissertação (Mestrado em Nefrologia) - Universidade Paulista, Recife, 2012.

DAHBOUR, S. S.; WAHBEH, A. M.; HAMDAN, M. Z. Mini mental status examination (MMSE) in stable chronic renal failure patients on hemodialysis: the effects of hemodialysis on the MMSE score: a prospective study. Hemodialysis International, Amá, v. 13, n. 1, p. 80-85, 2009. http://dx.doi.org/10.1111/j.15424758.2009.00343.x. PMid:19210282.

GINDRI, G. et al. Métodos em reabilitação neuropsicológica. In: FERNANDEZ, J. L.; FUKUSIMA, S. S. Métodos em Neurociência. São Paulo: Manole, 2012. p. 343-375.

ISER, B. P. M. et al. Fatores de risco e proteçáo para doenças crônicas não transmissíveis obtidos por inquérito telefônico - VIGITEL Brasil - 2009. Revista Brasileira de Epidemiologia, Porto Alegre, v. 14, n. 1, p. 90-102, 2011. http://dx.doi.org/10.1590/S1415$-790 X 2011000500010$.

JASSAL, S. V. et al. Differencial impairment of psychomotor efficiency and processing speed in patients with chronic Kidney disease. International Urology and Nephrology, Salford, v. 40, n. 3, p. 849-854, 2008. http://dx.doi.org/10.1007/s11255-008-9375-2. PMid:18443915.

KUSUMOTA, L. Avaliação da qualidade de vida relacionada à saúde de pacientes em hemodiálise. 2005. 144 f. Tese (Doutorado em Enfermagem) - Universidade de São Paulo, Ribeirão Preto, 2005.

LIMA, F. F. et al. Avaliação funcional pré e pós-programa de exercício físico de pacientes em hemodiálise. $M e-$ dicina, Ribeirão Preto, v. 46, n. 1, p. 24-35, 2013.

LOUREIRO, F. M. et al. Perfil de pacientes com insuficiência renal crônica, atendidos na unidade de hemodiálise de Linhares - ES. Enciclopédia Biosfera Centro Cientifico Conhecer, Espírito Santo, v. 13, n. 7, p. 15061513, 2011.

MADERO, M.; GUL, A.; SARNAK, M. J. Cognitive function in chronic kidney disease. Seminars in Dialysis, Boston, v. 21, n. 1, p. 29-37, 2008. http:// dx.doi.org/10.1111/j.1525-139X.2007.00384.x. PMid:18251955.

MATOS, E. F.; LOPES, A. Modalidades de hemodiálise ambulatorial: breve revisão. Acta Paulista de 
Enfermagem, Campinas, v. 22, p. 569-571, 2009. (Número Especial). http://dx.doi.org/10.1590/S010321002009000800025.

MORSCH, C.; GONÇALVES, L. F.; BARROS, E. Índice de gravidade da doença renal, indicadores assistenciais e mortalidade em pacientes em hemodiálise. Revista da Associação Médica Brasileira, São Paulo, v. 51, n. 5, p. 296-300, 2005. http://dx.doi.org/10.1590/S010442302005000500023 .

MURRAY, A. M. Cognitive impairment in the aging dialysis and chronic kidney disease populations: an occult burden. Advances in Chronic Kidney Disease, Minneapolis, v. 15, n. 2, p. 123-132, 2008. http://dx.doi. org/10.1053/j.ackd.2008.01.010. PMid:18334236.

MURRAY, A. M. et al. Cognitive impairment in hemodialysis patients is common. Neurology, Minneapolis, v. 67, n. 2, p. 216-223, 2006. http:// dx.doi.org/10.1212/01.wnl.0000225182.15532.40. PMid:16864811.

NIEDERAUER, M. Número de filhos é maior quanto menor é a escolaridade da mulher. Correio Braziliense, Brasília, 28 nov. 2012. Disponível em: <http://www. correiobraziliense.com.br/app/noticia/eu-estudante/ensino_educacaobasica/2012/11/28/ensino_educacaobasica_interna,336277/numero-de-filhos-e-maior-quanto-menor-e-a-escolaridade-da-mulher.shtml>. Acesso em: 23 jul. 2014.

OLIVEIRA, M. P. et al. Trabalho e qualidade de vida relacionada à saúde de pacientes em diálise peritoneal. Acta Paulista de Enfermagem, São Paulo, v. 25, n. 2, p. 352-357, 2012. http://dx.doi.org/10.1590/S010321002012000300006 .

PAULO, D. L. V.; YASSUDA, M. S. Queixas de memória de idosos e sua relação com escolaridade, desempenho cognitivo e sintomas de depressão e ansiedade. Archives of Clinical Psychiatry, Sáo Paulo, v. 37, n. 1, p. 23-26, 2010. http://dx.doi.org/10.1590/S010160832010000100005 .

PEREIRA, A. A. et al. Subcortical cognitive impairment in dialysis patients. Hemodialysis International, Boston, v. 11, n. 3, p. 309-314, 2007. http://dx.doi.org/10.1111/ j.1542-4758.2007.00185.x. PMid:17576295.

QUEIROZ, L. O.; NASCIMENTO, R. G. Repercussöes da hemodiálise na função respiratória de pacientes portadores de Insuficiência renal crônica. 2006. 91 f. Monografia (Trabalho de Conclusão de Curso em Fisioterapia) Universidade da Amazônia, Belém, 2006.

RADIC, J. et al. The possible impact of dialysis modality on cognitive function in chronic dialysis patients. The Netherlands Journal of Medicine, Haarlem, v. 68, n. 4, p. 153-157, 2010. PMid:20421655.

REIS, L. A. et al. Déficit cognitivo como fator de risco para a limitação de atividades cotidianas em idosos institucionalizados. Revista de Psicologia, Fortaleza, v. 2, n. 1, p. 126-136, 2011.
RIELLA, M. C. Princípios de nefrologia e distúrbios hidroeletrolíticos. Rio de Janeiro: Guanabara Koogan, 2010.

SCHRAIBER, L. B.; GOMES, R.; COUTO, M. T. Homens e saúde na pauta da saúde coletiva. Ciência \& Saúde Coletiva, São Paulo, v. 10, n. 1, p. 7-17, 2005. http:// dx.doi.org/10.1590/S1413-81232005000100002.

SERVIÇO BRASILEIRO DE APOIO ÀS MICRO E PEQUENAS EMPRESAS - SEBRAE. Perfil do produtor rural. São Paulo, 2012. (Série Estudos e Pesquisas). Disponível em: <http://www.sebrae.com.br/ Sebrae/Portal\%20Sebrae/Anexos/perfil_do_produtor_ rural_-2012_.pdf>. Acesso em: 23 jul. 2014.

SILVA, J. A. V. Relaçâo entre comorbidades e declinio cognitivo leve em pacientes clinicos com idade maior que 50 anos na Cidade de Santana do livramento, RS. 2011. 103 f. Dissertação (Mestrado em Gerontologia Biomédica) Pontífica Universidade Católica do Rio Grande do Sul, Porto Alegre, 2011.

SILVA, S. T. et al. Capacidade cognitiva de indivíduos com doença renal crônica: relação com características demográficas e clínicas. Jornal Brasileiro de Nefrologia, Viçosa, v. 36, n. 2, p. 163-170, 2014. http://dx.doi. org/10.5935/0101-2800.20140026.

SOCIEDADE BRASILEIRA DE NEFROLOGIA SBN. Censo de diálise 2013. São Paulo, 2013. Disponível em: <http://sbn.org.br/pdf/censo_2013_publico_leigo. pdf>. Acesso em: 24 jun. 2014.

SZUSTER, D. A. C. et al. Sobrevida de pacientes em diálise no SUS no Brasil. Cadernos de Saúde Pública, Belo Horizonte, v. 28, n. 3, p. 415-424, 2012. http://dx.doi. org/10.1590/S0102-311X2012000300002.

TAVARES, A. A. et al. (Re) Organização do cotidiano de indivíduos com doenças crônicas a partir da estratégia de grupo. Cadernos de Terapia Ocupacional da UFSCar, São Carlos, v. 20, n. 1, p. 95-105, 2012. http://dx.doi. org/10.4322/cto.2012.011

TEDESCO, S.; FERRARI, S. M. Acesso à teoria da técnica trilhas associativas. Revista do Centro de Estudos de Terapia Ocupacional, São Paulo, v. 3, n. 5, p. 32-36, 2000.

TYRRELL, J. et al. Older patients undergoing dialysis treatment: cognitive functioning, depressive mood and health-related quality of life. Aging \& Mental Health, Grenoble, v. 9, n. 4, p. 374-379, 2005. http://dx.doi. org/10.1080/13607860500089518. PMid:16019295.

VIEIRA, M. C. et al. Intervenção da Terapia Ocupacional junto a pacientes com doença renal crônica em tratamento hemodialítico: uma revisão. Revista do Hospital Universitário da UFMA, São Luís, v. 7, n. 1, p. 43-45, 2006.

ZAMBONATO, T. K.; THOMÉ, F. S.; GONÇALVES, L. F. S. Perfil Socioeconômico dos Pacientes com Doença Renal Crônica em Diálise na Região Noroeste do Rio Grande do Sul. Jornal Brasileiro de Nefrologia, Porto Alegre, v. 30, n. 3, p. 192-200, 2008. 


\section{Contribuição dos Autores}

Valeska e Karina participaram da coleta dos dados, redação do texto e organização das fontes. Milady e Katiuscia participaram da concepção do projeto de pesquisa, análise estatística e revisão crítica. Todos os autores aprovaram a versáo final do texto.

\section{Fonte de Financiamento}

O trabalho recebeu financiamento da Fundação de Amparo à Pesquisa e Desenvolvimento Científico do Maranhão (FAPEMA).

\section{Notas}

${ }^{1}$ Este estudo foi aprovado pelo Comitê de Ética e Pesquisa do Hospital Universitário da Universidade Federal do Maranhão (Parecer consubstanciado 05935612.7.0000.5086) e apresentado no X Congresso Norte Nordeste de Terapia Ocupacional em Belém/ PA. 